\title{
Stationary shocks in periodic highly nonlinear granular chains
}

\author{
Alain Molinari ${ }^{1}$ and Chiara Daraio ${ }^{2, *}$ \\ ${ }^{1}$ Laboratoire de Physique et Mécanique des Matériaux, Université Paul Verlaine-Metz, Ile du Saulcy, Metz, 57045, France \\ ${ }^{2}$ Graduate Aerospace Laboratories (GALCIT) and Applied Physics, California Institute of Technology, Pasadena, \\ California 91125, USA \\ (Received 26 May 2009; published 17 November 2009)
}

\begin{abstract}
We study the existence of stationary shock waves in uniform and periodic heterogeneous highly nonlinear granular chains governed by a power-law contact interaction, comparing discrete and continuum approaches, as well as experiments. We report the presence of quasisteady shock fronts without the need for dissipative effects. When viscous effects are neglected, the structure of the leading front appears to be solely the result of dispersive effects related to the lattice wave dispersion and, for heterogeneous bead chains, to the impedance mismatch between material domains. We report analytically and numerically the shock-width scaling with the variation in the particles periodicity (cell size) and compare the obtained results with experiments. We check the state $(-)$ behind the shock front via quasistatic compression analysis and report a very good agreement between theory and numerical data.
\end{abstract}

DOI: 10.1103/PhysRevE.80.056602

PACS number(s): 45.70. -n, 05.45.Yv, 46.40.Cd, 43.25.+y

\section{INTRODUCTION}

The problem of wave propagation in heterogeneous nonlinear media has received a lot of attention, see for instance Whitham [1] and Remoissenet [2]. The case of heterogeneous structures with spatial periodicity is of special interest by offering a wide range of applications in the field of composite materials (laminates or fiber composites) [3-9]. Onedimensional lattices of granular media (highly nonlinear particle chains) have been widely recognized in the literature as the birthplace of the highly nonlinear wave theory for uniform and heterogeneous periodic systems [10-13]. One of their most notable characteristics is the ability to support the formation and propagation of compact solitary waves characterized by a finite wavelength and a nonlinear amplitudevelocity scaling. Recently, these systems have been receiving increasing attention as they represent one of the most experimentally and numerically tractable platforms for the study of the interplay between discrete and continuum approaches [14]. In addition, the formation and propagation of highly nonlinear pulses in uniform and heterogeneous media has allowed the discovery of interesting physical phenomena like pulse energy trapping [15-17], anomalous reflections [18], and tunable acoustic wave propagation [19].

In this paper, the case of granular materials with periodic heterogeneity is considered. To simplify the analysis without much restriction on the physics of the problem, we analyze a one-dimensional chain of particles with uniform diameters, in elastic contact with each other. The nonlinear response of a chain of spherical particles has been extensively analyzed both on theoretical and experimental aspects [11-14,20-29]. One of the interesting aspects of these systems stems from the collective particles behavior: although the materials in each individual bead are assumed to have a linear elastic response, the ensemble of particles (granular chain) has a

\footnotetext{
*Author to whom correspondence should be addressed; daraio@caltech.edu
}

highly nonlinear response which stems from the Hertzian contact interaction in compression and from the zero tensile response.

The response of a bead chain to the impact of a bead of finite mass at an extremity was analyzed $[11,30]$. It was shown that this impact generates solitary waves in increasing number depending on the striker mass and/or the duration of the exciting pulse. Less attention was accorded to the shock wave generated by a constant velocity applied to an extremity of the bead chain (for instance by the impact of a long projectile). When viscous dissipation is present, Herbold and Nesterenko have shown that a stationary shock front can be formed in a uniform chain of particles [26], and that a steady shock front propagates with a constant velocity and a constant shape. These authors investigated the structure of the shock front analytically.

The scope of this paper is to analyze the structure of the shock front generated in periodic heterogeneous chains of spherical particles by the application of a constant velocity at an extremity of the chain. While the existence of a steady shock front has gained some attention for uniform bead chains, no studies have been developed on the shock-wave structure in the case of periodic heterogeneous systems. Understanding the propagation properties of shock structures in granular media is particularly relevant for the development of new shock-protecting systems, energy trapping, and blast mitigation devices based on highly nonlinear dynamics. For example, it is interesting to learn how one can control the shock-front width as a function of the materials parameters and geometrical arrangement of the nonlinear system. Also, the presence of such "compact" shock front could facilitate the engineering design of systems for shock confinement and impulse redirection.

In this paper, we consider chains of particles composed by the periodic repetition of a basic unit cell made up of an arrangement of two types of beads (steel and polytetrafluoroethylene). The analysis is based on direct numerical simulations of the dynamic response of the discrete bead system, on analytical results obtained from homogenized equations and on experimental tests. A particular attention will be de- 
voted to the leading shock front when no dissipation is taken into account. The relationship between the microstructure of the bead chain (i.e., the periodic cell pattern) and the structure of the shock front will be investigated and a scaling law will be found. We note that together with nonlinear effects, dispersive effects do play a primary role in the shaping of the shock-wave structure. Two types of dispersive effects are involved: those related to the impedance mismatch between beads of different properties and those related to the discrete nature of the bead chain.

The paper is organized as follows: in Sec. II we describe the materials configurations used in the analysis, the numerical approach followed, and the experimental set up. We then introduce in Sec. III the basic continuum equations describing the traveling waves in periodic heterogeneous granular chains. We subsequently present in Sec. IV the numerical results for the presence of shock waves in the periodic composite chains of particles. In Sec. V we develop a theoretical framework for the analysis of the quasisteady shock waves. Their structure is studied by analytical means in Sec. VI for the case of uniform and diatomic (periodic repetition of twoparticle unit cells) chains. We check in Sec. VII the consistency of our approaches by comparing directly the two analytical approaches followed and by directly matching experiments, numerical, and theoretical frameworks. Finally, in Sec. VIII, we demonstrate the existence of a scaling law (linear relationship) between the width of the steady shock front and the fundamental cell size in the system. Conclusions are drawn in Sec. IX, and possible applications of this work are foreseen.

\section{MATERIAL CONFIGURATION}

We consider the dynamic response of a one-dimensional bead chain made up of a regular alignment of beads. The chain is assumed to be either uniform or to be composed of a periodic distribution of unit cells. Fundamental unit cells composed of two different types of beads are called dimers. We refer to a dimer chain as a one-dimensional granular system of different lengths with periodic repetition of dimers. Dimers considered in this paper are made up of a series of $N_{1}$ aligned beads of type 1 followed by a series of $N_{2}$ beads of type 2 . The corresponding chain is denoted as $\left(N_{1}: N_{2}\right)$ dimer. Examples of the possible granular configurations studied are shown in Fig. 1. Beads (1) and (2) have respectively masses $m_{1}$ and $m_{2}$, Young's moduli $E_{1}$ and $E_{2}$, Poisson coefficients $\nu_{1}$ and $\nu_{2}$, and initial radii $R_{1}$ and $R_{2}$ (diameters $D_{1}$ and $D_{2}$ ). For instance in Fig. 1(c) the bead chain is a $(3: 2)$ dimer. The bead on the left boundary of the chain is assumed to be always of type (1). In the applications considered in this paper, (1) and (2) designate respectively stainless-steel beads and PTFE (polytetrafluoroethylene) beads. The material characteristics and geometry of these beads are given in Table I.

We denote by $\lambda$ the average stretch over a unit cell subjected to a quasistatic compressive force $F$. This stretch is identical for all unit cells and equal to the overall stretch of the chain. The overall quasistatic response of the chain of particles is defined by the relationship between $F$ and $\lambda$. (a)
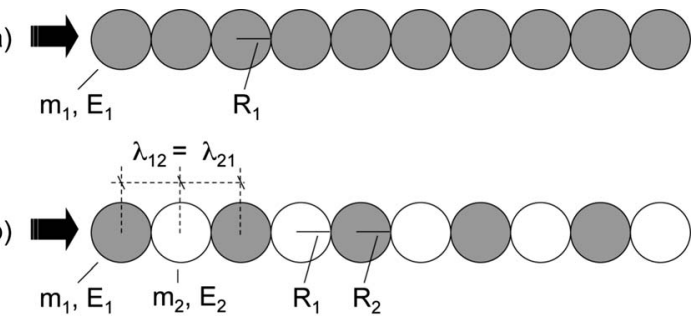

(c)

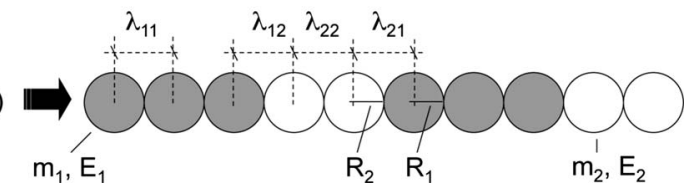

FIG. 1. Schematic representation of the bead chains: (a) uniform bead chain, (b) (1:1)-dimer chain, (c) (3:2)-dimer chain.

First, consider the case of a $(1: 1)$ dimer as defined in Fig. 1(b). The initial distance between the centers of two neighbor beads is $L_{0}=\left(D_{1}+D_{2}\right) / 2$. This distance is changed into $L$ after application of the force $F$ and the stretch is $\lambda=L / L_{0}$. The variation $\delta$ (taken as positive) of the distance between the centers of neighbor bead is obtained in terms of $F$ by the Herztian contact theory,

$$
\begin{gathered}
F=\frac{4}{3} E^{*} \sqrt{R^{*}} \delta^{k}, \\
k=3 / 2, \quad \frac{1}{E^{*}}=\frac{1-\nu_{1}^{2}}{E_{1}}+\frac{1-\nu_{2}^{2}}{E_{2}}, \quad \frac{1}{R^{*}}=\frac{1}{R_{1}}+\frac{1}{R_{2}} .
\end{gathered}
$$

Considering that $\delta=L_{0}-L=L_{0}(1-\lambda)$, the quasistatic response (1) can be written as

$$
F=F_{Q S}(\lambda)=K(1-\lambda)^{k} .
$$

For $k=3 / 2$, we have:

$$
K=\frac{1}{3} E^{*} \sqrt{D_{1} D_{2}}\left(D_{1}+D_{2}\right) .
$$

A uniform bead chain is treated as a particular case of the above equations.

The effective response $F_{Q S}(\lambda)$ of the composite bead chain is derived in Appendix A for the general case where $N_{1} \geq 1$ and $N_{2} \geq 1$. The result has the same form as Eq. (3a),

$$
F=F_{Q S}(\lambda)=K_{e f f}(1-\lambda)^{k},
$$

with the overall stretch $\lambda$ being given by

TABLE I. Materials' properties [19].

\begin{tabular}{lcccc}
\hline \hline Material & $\begin{array}{c}\text { Mass } \\
(\mathrm{g})\end{array}$ & $\begin{array}{c}E \\
(\mathrm{GPa})\end{array}$ & $\begin{array}{c}\text { Poisson ratio } \\
(v)\end{array}$ & $\begin{array}{c}\text { Radius } \\
(\mathrm{mm})\end{array}$ \\
\hline Stainless steel & 0.45 & 193 & 0.30 & 2.38 \\
PTFE & 0.123 & 1.46 & 0.46 & 2.38 \\
\hline \hline
\end{tabular}




$$
\lambda=f_{11} \lambda_{11}+f_{22} \lambda_{22}+f_{12} \lambda_{12} .
$$

The effective stiffness modulus $K_{\text {eff }}$ is defined by Eq. (A14) in Appendix A. The coefficients $f_{11}, f_{22}$, and $f_{12}$ are also defined in Appendix A. $\lambda_{12}$ is the stretch between the centers of two neighbor beads (1) and (2) as illustrated in Figs. 1(b) and 1(c). Note that $\lambda_{12}=\lambda_{21}$. The value of $\lambda_{12}$ is identical to $\lambda$ defined by (3a-b). $\lambda_{11}\left(\lambda_{22}\right)$ is the stretch between the centers of two neighboring beads (1) [(2)], see Fig. 1(c), and is given in Appendix A

For non-Hertzian contact we have $k \neq 3 / 2$. For instance, for the contact of a cone with a flat surface we have $k=2$ [31]. For a dimer chain made of aligned cones of types (1) and (2), the result (3) will remain valid with $k=2$ and an appropriate value of $K$. Only Hertzian contact (spherical beads) will be considered in this paper; however some results can be extended to a generalized contact interaction law with a non-Hertzian exponent.

\section{A. Numerical analysis}

Numerical simulations are conducted by using the numerical scheme described in $[13,32]$. We represent the elastic particles composing the chains by point masses (concentrated at the bead's centers) connected by nonlinear springs, accounting for the Hertzian contact interactions. We solve a discrete system of equation for the particles equation of motion by using the Runge-Kutta integration method [32]. The time step used in the simulations was $5 \times 10^{-7} \mathrm{~s}$.

\section{B. Experimental setup}

One-dimensional chains of particles were assembled from different periodic combinations of stainless-steel beads (316type), with diameter $D=4.76 \mathrm{~mm}$ and mass $0.45 \mathrm{~g}$, and PTFE (polytetrafluoroethylene) particles of equal diameter and mass $0.123 \mathrm{~g}$ (all beads provided by McMaster-Carr). The systems were positioned vertically, and the alignment of the particles was ensured by using a 4-garolite rod holder as shown in Fig. 1 of [13]. Pulses of different durations and amplitudes were generated by impacting a long stainlesssteel cylindrical rod $(4.76 \mathrm{~mm}$ in diameter, mass equal to 33 $\mathrm{g}$ ) on the top particle of the chain (always selected to be a stainless-steel bead) from different heights. Two calibrated piezosensors $\left(\mathrm{RC} \sim 10^{3} \mu \mathrm{s}\right)$ were embedded inside selected particles [13] and connected to a Tektronix oscilloscope to monitor the force-time response of the system and obtain a precise measurement of the speed of signal propagation between particles.

\section{CONTINUUM APPROACH FOR TRAVELING WAVES IN CHAINS OF DIMERS}

We consider in this section the particular case of a $(1: 1)$ dimer. The unit cell is solely composed of two beads (1) and (2). In addition we assume that the beads have same diameter $D$. For long waves, a "homogenized" continuous response of the dynamic behavior of the bead chain can be obtained from the discrete formulation. We refer to [11] for a general presentation of the homogenization process, and summarize the results obtained in $[12,13]$ for a chain of $(1: 1)$ dimers.

The position of a material particle in the reference and the current configurations is, respectively, defined by $X$ and $x$. The longitudinal displacement is $u=x-X$ and the deformation gradient is $u_{x}=\partial u / \partial x$. The wave equation of the homogenized system can be written as $[12,13]$

$$
u_{\tau \tau}=u_{x}^{k-1} u_{x x}+G u_{x}^{k-3} u_{x x}^{3}+H u_{x}^{k-2} u_{x x} u_{x x x}+I u_{x}^{k-1} u_{4 x} .
$$

In this equation the normalized time $\tau$ is related to the physical time $t$ by

$$
\tau=t \sqrt{\frac{2 k D^{k+1} A}{m_{1}+m_{2}}},
$$

with

$$
A=\frac{2 E_{1} E_{2} D^{2-k}}{3\left[E_{1}\left(1-\nu_{2}^{2}\right)+E_{2}\left(1-\nu_{1}^{2}\right)\right]} .
$$

Here $k$ represents the nonlinear contact exponent in the force-displacement relationship; $E_{1}$ and $E_{2}$ are the elastic moduli of the particles (1) and (2), respectively, and $v_{1}$ and $v_{2}$ represent the Poisson's ratios. Note that the normalized time $\tau$ has the dimension of a length.

Other parameters in Eq. (5) are defined by

$$
\begin{gathered}
G=\frac{1}{6}\left(2-3 k+k^{2}\right) D^{2} \frac{m_{1}^{2}}{\left(m_{1}+m_{2}\right)^{2}}, \\
H=2(k-1) D^{2} \frac{2 m_{1}^{2}+m_{1} m_{2}-m_{2}^{2}}{6\left(m_{1}+m_{2}\right)^{2}}, \\
I=2 D^{2} \frac{m_{1}^{2}-m_{1} m_{2}+m_{2}^{2}}{6\left(m_{1}+m_{2}\right)^{2}} .
\end{gathered}
$$

The lack of symmetry with respect to indices 1 and 2, as in Eq. (8), indicates that the solution is only approximate. However, when compared against experiments and direct numerical simulations, these results have a good predictivity $[12,13]$. In the present paper, we compare direct numerical simulation of the discrete system with theoretical considerations, and show that the model defined by Eqs. (5)-(10) provides good results if the condition $m_{1} \geq m_{2}$ is satisfied.

We seek traveling-wave solutions of the Eq. (5) that have the following form:

$$
u=u(\xi) \quad \text { with } \quad \xi=x-V_{S} \tau .
$$

The rescaled (nondimensional) wave speed $V_{S}$ is related to the physical speed $\hat{V}_{S}$ by

$$
V_{S}=\hat{V}_{S} t / \tau=\hat{V}_{S} \sqrt{\frac{m_{1}+m_{2}}{2 k D^{k+1} A}} .
$$

The following definition is introduced:

$$
z=\left(-u_{x}\right)^{1 / p}
$$

where $-u_{x}$ is assumed to be positive. The exponent is defined by 


$$
p=\left[(H+3 I)-\sqrt{(H+3 I)^{2}-8 I(G+H+I)}\right][2(G+H+I)]^{-1} .
$$

Note that there are two possible values of $p$ that lead to this reduction, and we chose one arbitrarily to simplify the resulting ordinary differential equation. Then, Eq. (5) takes the form [13]

$$
\frac{d^{2} z}{d \xi^{2}}=\mu z^{\eta}-\sigma z
$$

with parameters defined by

$$
\begin{gathered}
\eta=1+p-k p, \\
\sigma=\frac{1}{I(k p+a)}, \\
\mu=\frac{V_{S}^{2}}{I(p+a)}, \\
a=1-k p+3(p-1)+p H / I .
\end{gathered}
$$

Introducing the stretch $\lambda=\partial x / \partial X$, it follows from Eq. (13) that

$$
z=(1-\lambda)^{1 / p} .
$$

The analysis is limited to small deformations; therefore there is no necessity to distinguish Lagrangian and Eulerian coordinates of a material particle.

Note that $\sigma$ scales as $1 / D^{2}$ and is a function of $k$ and of $m_{1} / m_{2}$,

$$
\sigma=\frac{1}{D^{2}} f\left(k, m_{1} / m_{2}\right)
$$

Similarly

$$
\begin{gathered}
\mu=\frac{V_{S}^{2}}{D^{2}} g\left(k, m_{1} / m_{2}\right), \\
\eta=\eta\left(k, m_{1} / m_{2}\right) .
\end{gathered}
$$

In the case of uniform beads it follows from Eqs. (8)-(10), (14), and (16)-(19) that

$$
\begin{gathered}
I=\frac{D^{2}}{12}, \quad G=\frac{1}{24}\left(2-3 k+k^{2}\right) D^{2}, \quad H=\frac{1}{6}(k-1) D^{2}, \\
p=\frac{2}{k+1}, \quad a=0, \quad \sigma=\frac{6}{D^{2}} \frac{k+1}{k}, \\
\eta=\frac{3-k}{1+k} \quad \mu=\frac{6(k+1) V_{S}^{2}}{D^{2}} .
\end{gathered}
$$

For Hertzian contact, we have $k=3 / 2$ and it follows:

$$
\sigma=\frac{10}{D^{2}} \quad \eta=\frac{3}{5} \quad \mu=\frac{10 \hat{V}_{S}^{2}}{D^{2} c^{2}},
$$

where:

$$
c^{2}=\frac{2 E}{\pi \rho_{0}\left(1-\nu^{2}\right)}\left(\rho_{0} \text { mass density of the bead }\right) .
$$

For uniform beads and Hertzian contact, the relationship (12) between the physical velocity $\hat{V}_{S}$ and the nondimensional velocity $V_{S}$ reads

$$
\hat{V}_{S} / V_{S}=c \sqrt{\frac{3}{2}} .
$$

\section{NUMERICAL INVESTIGATION OF QUASISTEADY SHOCK WAVES IN PERIODIC HETEROGENEOUS BEAD CHAINS}

We consider here shock waves generated by the application of a constant velocity $v^{-}$at the left extremity of a chain of beads. It is well known that steady wave fronts result in general from the competition between the front steepening due to the nonlinearity of the system's response and the front widening due to dissipative and dispersive effects. Dispersive effects are related to the discrete aspect of the bead chain on one hand. This is a well-known phenomenon in lattice crystals. On the other hand, for heterogeneous bead chains (beads made up of different materials) additional dispersive effects are produced by wave reflections and refractions due to impedance mismatch between beads of different material properties.

Shock waves in uniform bead chains have been investigated by different authors [11,24-27,33-36]. The relative role of dissipative and dispersive effects on the shock-front structure is not well understood. Even when dissipative effects are absent, the shock front can have a steady structure. For instance, Hill and Knopoff [37] have observed in their numerical experiments on strong shocks in one-dimensional uniform Toda lattices initially at rest that the shock is led by a soliton. The same observation was made by Nesterenko for uniform bead chains [11]. The leading hump of the shock wave was reported to be very close to a stationary solitary wave propagating at the head of a globally nonstationary wave. The spatial distribution of the particle velocity was such that at the wave front, the particle velocity is raised from zero (state of rest) to a maximum value that is about twice the applied velocity $v^{-}$. Behind this shock front, the particle velocity shows oscillations of decaying amplitude and finally tends asymptotically to $v^{-}$far away the wave front. The velocity of the leading front was also reported to be equal to the solitary wave velocity with very good accuracy [11].

In this work we study numerically the structure of the shocks forming in chains in which periodic heterogeneities are considered and dissipative effects are disregarded. In the analysis all the particles in the chains are assumed to have identical diameters. A shock wave is generated by the impact of a massive striker at one extremity of the chain and the 

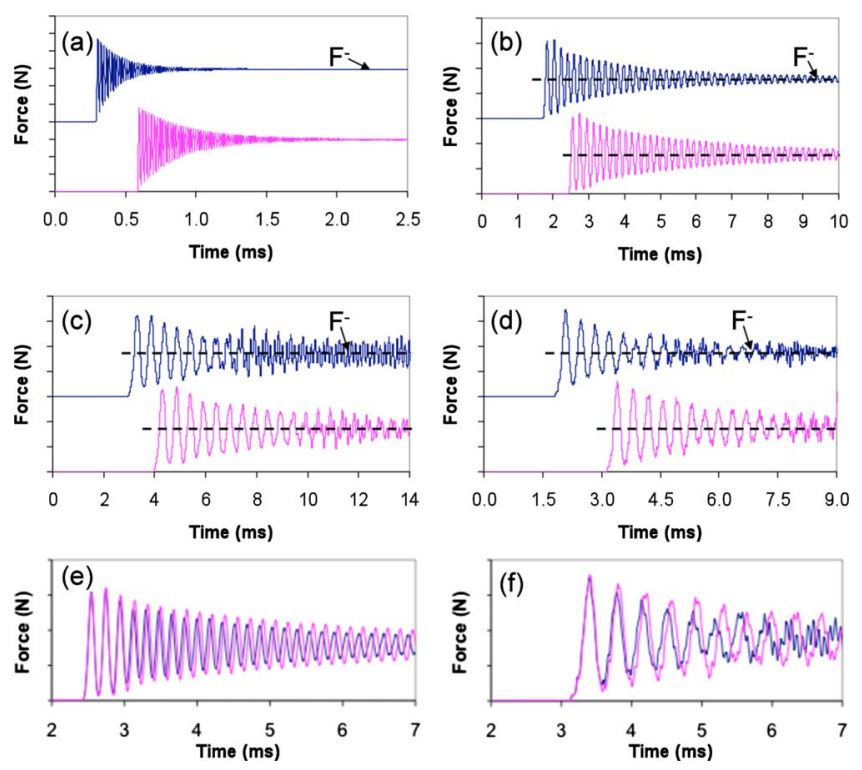

FIG. 2. (Color online) Numerical results showing the evolution with time of the compressive force $F$ at various bead locations for a given impact velocity $v^{-}(1 \mathrm{~m} / \mathrm{s})$. Four configurations for chains of 300 particles are considered: (a) Uniform chain, the top (dark) curve corresponds to particle 51 and the bottom (light) one to particle $101, Y$-axis scale is $25 \mathrm{~N}$. (b) (1:1) dimer chain, the top curve corresponds to particle 51 and the bottom one to particle 71, $Y$-axis scale is $5 \mathrm{~N}$. (c) (3:3) dimer chain, the top curve corresponds to particle 95 and the bottom one to particle $125, Y$-axis scale is $5 \mathrm{~N}$. (d) $(5: 1)$ dimer chain, the top curve corresponds to particle 90 and the bottom one to particle $150, Y$-axis scale is $10 \mathrm{~N}$. In (a)-(d) the curves have been shifted on the vertical axis to ease the visualization. For all cases, the force first rises to a local peak value $\boldsymbol{F}_{\text {max }}$ and then sustains oscillations with decreasing amplitude. The force is tending asymptotically to the limit $F^{-}$. Note that an accurate estimate of $F^{-}$can be obtained by considering the time average of the force over some oscillations (first oscillation disregarded). (e) Curves from panel (b) in which the signal from particle 51 has been superimposed to particle 71 for comparison. (f) Curves from panel (d) in which the signal from particle 90 has been superimposed to particle 150 for comparison.

existence of quasisteady shock waves is illustrated. The striker's impact velocity is denoted by $v^{-}$. It is reported in $[11,27]$ that the first oscillation in the force-time diagram (Fig. 2) should be very close to a solitary wave in the case of small dissipation. It is then deduced that $F_{\max } / F^{-}=1.95$. This relationship is well satisfied in our simulations.

The results presented in Fig. 2 show that almost identical time histories for the leading fronts and initial oscillations are obtained when recording the force at "homologous" beads separated by the distance $n L_{\text {cell }}$, where $L_{\text {cell }}$ is the cell size and $n$ is a positive integer. For instance, in Fig. 2(d), the particles no. 90 and no. 150 are at ten cell sizes from each other. Note that for nonhomologous beads no. 90 and no. 151 the time histories of the force would be different. To clarify the comparison, Figs. 2(e) and 2(f) show results for the (11)-dimer and the (5-1)-dimer in which the signal of the particle closest to the impact has been shifted to overlap with the signal recorded from the homologous points. It is evident that the fronts and the following leading pulses overlap ex- actly, but after few oscillations the tails tend to go out of phase.

The shock front is characterized by half of the first oscillation, where the force rises to the maximum value $\boldsymbol{F}_{\text {max }}$. The velocity $\hat{V}_{S}$ of the wave front can be estimated by considering the arrival time of the peak value of the force at two homologous bead centers. As soon as the shock front is at few cell sizes from the chain extremity, the shock velocity is observed to have a constant value.

From these considerations it can be concluded that, for an observer moving with the shock velocity $\hat{V}_{S}$, the wave pattern is not changed when the observer arrives at homologous beads (distant by an integer numbers of cells). When the observer takes a different position in the unit cell the wave pattern is changing, but due to the periodicity of the chain structure, the observer experiences the periodicity $T$ $=L_{\text {cell }} / \hat{V}_{S}$ in the time evolution of the wave pattern. For the observer, time averaging will define a steady shock-wave structure propagating with the velocity $\hat{V}_{S}$. These considerations can be summarized as follows. In general the force $F=F(x, t)$ is function of position and time. Considering the coordinate $\xi=x-\hat{V}_{S} t$, the wave pattern has the form $F$ $=\widetilde{F}(\xi, t)$ for an observer moving with the shock-wave velocity $\hat{V}_{S}$. Due to the spatial periodicity of the bead-chain structure, $\widetilde{F}(\xi, t)$ has the time periodicity $T=L_{\text {cell }} / \hat{V}_{S}$. We shall qualify this wave pattern as a quasisteady shock front. A steady wave can be defined by time averaging of the quasisteady wave over a period,

$$
F_{s t}(\xi)=\frac{1}{T} \int_{t_{0}}^{t_{0}+T} \tilde{F}(\xi, t) d t .
$$

As discussed in the foregoing, $\hat{V}_{S}$ and $F^{-}$can be obtained in terms of the impact velocity $v^{-}$from the numerical simulations of the dynamic response of a bead chain. The force $F^{-}$ corresponds to the steady state $\left({ }^{-}\right)$reached far behind the shock front. The stretch $\lambda^{-}$associated to this state is related to $F^{-}$by the constitutive law (4),

$$
F^{-}=F_{Q S}\left(\lambda^{-}\right)=K_{e f f}\left(1-\lambda^{-}\right)^{k} .
$$

The values of $\hat{V}_{S}$ and $F^{-}$are reported in Table II for different impact velocities comparing numerical and theoretical data (see next section).

\section{THEORETICAL ANALYSIS OF QUASISTEADY SHOCK FRONTS IN PERIODIC COMPOSITE BEAD CHAINS}

In this section, a theoretical framework is developed for the analysis of quasisteady shock waves. The laws relating $\hat{V}_{S}$ and $F^{-}$to the impact velocity $v^{-}$will be determined. These results are then compared to the data obtained by numerical means in the previous section. For a given impact velocity $v^{-}$the steady state $(-)$reached far behind the shock can be characterized by considering the following simplified framework. Note first that, to fulfill the small deformation hypothesis and the Hertzian contact theory, only moderate impact velocities are considered. In that context, "thermal 
TABLE II. Comparison of the numerical and analytical values obtained for the shock velocity $\left(\hat{V}_{S}\right)$, the force amplitude $\left(F^{-}\right)$and the front width for different impact velocities. Here we considered a (1:1) dimer chain composed of 300 particles, values are reported in reference to particles number 51 and 57 (PTFE) from the impacted side.

\begin{tabular}{|c|c|c|c|c|c|c|}
\hline \multicolumn{4}{|c|}{ Numerical data } & \multicolumn{3}{|c|}{ Analytical data } \\
\hline $\begin{array}{l}v^{-} \\
(\mathrm{m} / \mathrm{s})\end{array}$ & $\begin{array}{l}F^{-} \\
(N)\end{array}$ & $\begin{array}{c}\hat{V}_{s} \\
(\mathrm{~m} / \mathrm{s})\end{array}$ & $\begin{array}{c}w \\
(\mathrm{~mm})\end{array}$ & $\begin{array}{l}F^{-} \\
(N)\end{array}$ & $\begin{array}{c}\hat{V}_{s} \\
(\mathrm{~m} / \mathrm{s})\end{array}$ & $\lambda^{-}$ \\
\hline 0.1 & 0.50 & 84.50 & 16.48 & 0.4912 & 81.62 & 0.9988 \\
\hline 0.5 & 3.43 & 116.60 & 17.72 & 3.389 & 112.6 & 0.9956 \\
\hline 1 & 7.85 & 134.72 & 18.46 & 7.786 & 129.3 & 0.9923 \\
\hline 10 & 124.00 & 211.56 & 18.40 & 123.4 & 205.0 & 0.9512 \\
\hline 25 & 375.50 & 255.00 & 18.87 & 370.5 & 246.2 & 0.8985 \\
\hline 50 & 860.40 & 292.92 & 19.63 & 851.3 & 282.9 & 0.8232 \\
\hline 100 & 1974.00 & 336.00 & 18.31 & 1956 & 324.9 & 0.6922 \\
\hline
\end{tabular}

effects" characterized by random and chaotic bead vibrations are negligible.

Ahead and far behind the shock front, the bead chain is respectively in the state of rest $(+)$ and in the state $(-)$ of quasistatic compression. In a first step, the shock is considered as a moving discontinuity across, which the particle velocity, the stretch, and the stress sustain an instantaneous jump from (+) to (-). For shocks of moderate amplitude, the jump relations will be obtained by taking the quasistatic response given by Eq. (4) as the Hugoniot ("thermal effects" neglected). To characterize the shock-wave structure, i.e., the layer across which the physical variables evolve from state $(+)$ to state $(-)$, additional information about the bead-chain response is necessary. This problem will be addressed in the next section in the case of uniform bead chains and $(1: 1)$ dimers.

States (-) and (+) are related by jump relationships obtained from the conservation of momentum and the compatibility condition. The law of conservation of momentum, in the direction $x$ of wave propagation, has the form

$$
\frac{\partial \hat{F}}{\partial x}=\hat{\rho}_{0} \frac{\partial v}{\partial t}
$$

where $\hat{F}$ is the longitudinal force, $v$ is the particle velocity, and $\hat{\rho}_{0}$ is the mass per unit chain length. Note that by definition the compressive force $F$ (positive under compression) is

$$
F=-\hat{F}
$$

The compatibility equation reads

$$
\frac{\partial v}{\partial x}=\frac{\partial \lambda}{\partial t}
$$

The stretch $\lambda$ is given in terms of the longitudinal displacement $u$ by

$$
\lambda=1+\frac{\partial u}{\partial x} .
$$

For a steady wave and for an observer moving with the shock velocity $\hat{V}_{S}$, the variables are solely function of $\xi=x$ $-\hat{V}_{S} t$. Then Eqs. (30) and (32) can be written as ordinary differential equations with respect to $\xi$. Upon integration of these equations, the following jump relationships are obtained:

$$
\begin{aligned}
& F^{-}-F^{+}=\hat{\rho}_{0} \hat{V}_{S}\left(v^{-}-v^{+}\right), \\
& v^{-}-v^{+}=-\hat{V}_{S}\left(\lambda^{-}-\lambda^{+}\right) .
\end{aligned}
$$

As the state $(+)$ is the initial state of rest, we have $F^{+}=0$, $\nu^{+}=0$, and $\lambda^{+}=1$. Thus Eqs. (34) and (35) take the form

$$
\begin{gathered}
F^{-}=\hat{\rho}_{0} \hat{V}_{S} v^{-}, \\
v^{-}=\hat{V}_{S}\left(1-\lambda^{-}\right) .
\end{gathered}
$$

By elimination of $\hat{V}_{S}$ between Eqs. (36) and (37) and by using the constitutive relationship (29), the stretch behind the shock can be calculated in terms of the impact velocity $v^{-}$,

$$
\left(\lambda^{-}-1\right) F_{Q S}\left(\lambda^{-}\right)+\hat{\rho}_{0}\left(v^{-}\right)^{2}=0 .
$$

This relationship provides an implicit equation that allows us to determine $\lambda^{-}$in terms of the impact velocity $v^{-}$. The shock velocity $\hat{V}_{S}$ is then obtained from Eq. (37).

Considering the law (4), we obtain from Eq. (38) an explicit relationship between $\lambda^{-}$and the impact velocity,

$$
\lambda^{-}=1-\left(\frac{\hat{\rho}_{0}}{K_{e f f}}\left(v^{-}\right)^{2}\right)^{1 /(1+k)} .
$$

The compressive force is

$$
F^{-}=K_{e f f}\left(1-\lambda^{-}\right)^{k}=K_{e f f}\left(\frac{\hat{\rho}_{0}}{K_{e f f}}\right)^{k /(1+k)}\left(v^{-}\right)^{2 k /(1+k)} .
$$

The Eqs. (39) and (40) characterize the material state (-) behind the shock front.

The shock velocity $\hat{V}_{S}$ is given by Eq. (37),

$$
\hat{V}_{S}^{2}=\frac{K_{e f f}}{\hat{\rho}_{0}}\left(1-\lambda^{-}\right)^{k-1}=\left(\frac{K_{e f f}}{\hat{\rho}_{0}}\right)^{2 /(1+k)}\left(v^{-}\right)^{[2(k-1)] /(k+1)} .
$$

For Herzian contact we have $k=3 / 2$, and $\hat{V}_{S} \propto\left(v^{-}\right)^{1 / 5}$. As noted in $[11,27]$ this nonlinear relationship between shock velocity and impact velocity is quite different from the linear law $\hat{V}_{S}=c^{+}+S v^{-}$usually obtained for steady plastic shocks in polycrystalline metals [38,39].

The results obtained from the continuum theory (40) and (41) are compared in Table II for a (1:1) dimer with the data obtained in Sec. IV by direct numerical simulation of the discrete bead system. A satisfactory agreement is observed between these approaches. It should be noted that the results would be slightly different for a $(N: N)$ dimer since the stiff- 
TABLE III. Comparison of the numerical and analytical values obtained for the shock velocity $\left(\hat{V}_{S}\right)$, the force amplitude $\left(F^{-}\right)$, and the front width for different impact velocities. Here we considered a (5:1) dimer chain composed of 300 particles, values are reported in reference to particle number 103 and 121 (PTFE) from the impacted side.

\begin{tabular}{|c|c|c|c|c|c|c|}
\hline \multicolumn{4}{|c|}{ Numerical data } & \multicolumn{3}{|c|}{ Analytical data } \\
\hline $\begin{array}{l}v^{-} \\
(\mathrm{m} / \mathrm{s})\end{array}$ & $\begin{array}{l}F^{-} \\
(N)\end{array}$ & $\begin{array}{c}\hat{V}_{s} \\
(\mathrm{~m} / \mathrm{s})\end{array}$ & $\begin{array}{c}w \\
(\mathrm{~mm})\end{array}$ & $\begin{array}{l}F^{-} \\
(N)\end{array}$ & $\begin{array}{c}\hat{V}_{s} \\
(\mathrm{~m} / \mathrm{s})\end{array}$ & $\lambda^{-}$ \\
\hline 0.1 & 1.09 & 136.00 & 54.26 & 1.10 & 132.9 & 0.9992 \\
\hline 0.5 & 7.41 & 187.89 & 56.37 & 7.62 & 183.3 & 0.9973 \\
\hline 1 & 17.83 & 216.91 & 54.01 & 17.50 & 210.6 & 0.9953 \\
\hline 10 & 280.4 & 342.72 & 54.50 & 277.3 & 333.7 & 0.9700 \\
\hline 25 & 838.2 & 411.92 & 55.60 & 832.6 & 400.8 & 0.9376 \\
\hline 50 & 1970 & 476.00 & 55.70 & 1913 & 460.4 & 0.8914 \\
\hline 100 & 4511 & 542.28 & 54.23 & 4395 & 528.9 & 0.8109 \\
\hline
\end{tabular}

ness $K_{\text {eff }}$ depends on $N$ [see Eqs. (A14) and (A8)]. For instance, for $N=6$ and $\nu^{-}=0.1 \mathrm{~m} / \mathrm{s}$ the results are $F^{-}$ $=0.54 \mathrm{~N}$ and $\hat{V}_{S}=90.5 \mathrm{~m} / \mathrm{s}$. Additional results are shown in the Table III for a $(5: 1)$ dimer.

\section{SHOCK STRUCTURE FOR UNIFORM BEAD CHAINS AND CHAINS OF (1:1) DIMERS: THEORETICAL ANALYSIS}

Numerical simulations presented in Sec. IV support the existence of quasistationary shocks in elastic bead chains. Here we investigate in details the shock structure for composite as well as for uniform chains. The shock structure is defined as the layer where the material sustains a continuous transition (oscillatory in our case) from state $(+)$ to state $(-)$ respectively ahead and behind the shock front. In dissipative materials, the shock structure results from the competition between the steepening of the wave front due to the nonlinearity of the material response and the smoothing due to dissipative effects. Dissipation is the result of viscous damping or thermal conduction. Note that viscous effects have been introduced by Herbold and Nesterenko [26] to obtain fully steady shock waves in uniform bead chains. However, these results have not been extended to heterogeneous bead chains.

In the numerical and theoretical treatment of the present paper, the materials composing the particles are assumed to have a perfectly linear elastic response. Accordingly, the contact interaction between particles is assumed to follow the nonlinear Hertz law. Thus, the quasisteady shock structure observed in the numerical results shown in Sec. IV must be attributed to a physical phenomenon different from dissipation. This is not the case for the experiments (shown in Sec. VII) in which the effects of dissipation are evident and confirm the results reported in [26]. Indeed, it is well known that a shock can be structured by the interplay of dispersive effects and nonlinear material effects. An example is given by the Korteweg-de Vries equation governing shallow water waves [40].

Wave dispersion is a known phenomenon in the dynamic response of uniform chains of particles. Lattice dispersion occurs when the wavelength is of the order of the separation distance between bead centers. In heterogeneous chains an additional agent of wave dispersion is due to the impedance mismatch between domains occupied respectively by beads (1) and (2), having different mechanical properties. Considering for example a $\left(N_{1}: N_{2}\right)$ dimer with $N_{1}$ and $N_{2}$ being large enough, one expects wave reflection and refraction at the interfaces (1)-(2) and (2)-(1). It is known from the theory of elastic waves in heterogeneous elastic bodies that wave dispersion is the result of wave reflection and refraction at material interfaces. This problem has been analyzed in great details $[41,42]$, in particular for multilayer periodic media [4].

This implies that for a heterogeneous chain of particles wave dispersion comprises in general two different aspects working at different length scales. The first aspect is lattice dispersion at the scale of the separation distance $D$ between bead centers. The second is impedance mismatch at the interfaces between the domains occupied by beads (1) and (2) ( $N_{1}$ and $N_{2}$ being large enough). The scale of this additional dispersive effect is the cell size $\left(N_{1}+N_{2}\right) D$. For small values of $N_{1}$ and $N_{2}$, the lattice scale (microscopic scale) and the cell scale (mesoscopic scale) can not be separated.

An important feature of the present paper relies on the detailed analysis of the relationship between wave dispersion and the structure of quasisteady shock waves in bead chains. Of particular interest is the effect of $N_{1}$ and $N_{2}$ in composite chains.

The shock-wave patterns exhibited in Fig. 2 show oscillations with decaying amplitudes. For a (1:1) dimer chain, the solution is governed by Eq. (15). In the phase plane $(z, \omega=d z / d \xi)$, the trajectories emanating from the state $(+)$ $(z=0, \omega=0)$ have the form

$$
\omega= \pm \sqrt{\frac{2 \mu}{\eta+1} z^{\eta+1}-\sigma z^{2}} .
$$

These trajectories are defined for $0 \leq z \leq z^{*}$, where $z^{*}$ $=(2 \mu /[\sigma(\eta+1)])^{1 /(1-\eta)}$.

The state $\left(z^{-}, \omega=0\right)$, corresponding to the state $(-)$ behind the shock, can not be reached by any of the trajectories defined by Eq. (42). Thus the oscillations observed numerically in the wave pattern cannot be described by Eq. (15). However, as shown in [26] the addition of viscosity allows one to define a trajectory connecting the states $(+)$ and $(-)$ defined on both sides of the shock. For small viscosities, the shock structure $z(\xi)$ shows decaying oscillations until the value $z^{-}$ is reached. For larger viscosities the transition from $z=0$ to $z^{-}$occurs without oscillations. It is still an open question to describe by analytical means the decaying oscillations shown in Fig. 2, while there is no viscosity in the problem. The failure of Eq. (15) in doing so may indicate that the underlying long wave hypothesis brings too much simplification in the physics of the problem. The role of numerical damping as a factor of attenuation should also be clarified. The decay 
of oscillations might be also connected to thermalization and the trend toward equipartition of the energy as observed in other situations $[15,17,32]$.

Our purpose is not to describe the whole pattern of oscillations, but rather to analyze the first ramp where the force is raised from $F=0$ to $F=F_{\max }$. We shall call this leading part of the shock wave the shock front. For a nondissipative uniform bead chain, the shock front is constituted by half a solitary wave as discussed by Nesterenko [11]. This result remains almost unchanged if a small dissipation is accounted for. In this section a theoretical analysis of the shock front is developed based on Eq. (15) for stationary traveling waves. This equation describes the motion of stationary traveling waves in uniform bead chains and (1:1) dimers. The comparison with numerical analysis indicates that Eq. (15) provides a good approximation of the shock front in $(1: 1)$ dimers. This is an extension of the observations of Nesterenko [11] made for a uniform bead chain. The analysis of the shock structure depends on the value of the exponent $\eta$ in Eq. (15). To consider a specific example, it can be noted that for uniform bead chains we have, according to Eq. (25), $1<\eta \leq 3$ for $1>k \geq 0, \eta=1$ for $k=1$, and $0<\eta<1$ for $3>k>1$. For Hertzian contact we have $k=\frac{3}{2}$ and $\eta=\frac{3}{5}$. In the following, we shall restrict our analysis to the case $0<\eta<1$. Equation (15) has the form

$$
\frac{d^{2} z}{d \xi^{2}}=g(z)
$$

with

$$
g(z)=\mu z^{\eta}-\sigma z(\mu>0, \sigma>0), \quad z=\left(-u_{x}\right)^{1 / p} .
$$

Defining

$$
z^{-}=\left(\frac{\sigma}{\mu}\right)^{1 /(\eta-1)}
$$

we have, $g\left(z^{-}\right)=0$. As a consequence of $0<\eta<1$, we note that $g(z)>0$ for $0<z<z^{-}$.

We seek a solution $\xi \rightarrow z(\xi)$ of Eq. (15) such that there exist $\xi^{-}$for which $z\left(\xi^{-}\right)=z^{-}$.

Multiplying both members of Eq. (43) by $d z / d \xi$, it follows after integration that

$$
\frac{1}{2}\left\{\left[\frac{d z}{d \xi}(\xi)\right]^{2}-\left[\frac{d z}{d \xi}(\xi)\right]^{2}\right\}=\hat{g}(z)
$$

with

$$
\hat{g}(z)=-\int_{z}^{z^{-}} g(u) d u=\frac{\mu}{\eta+1}\left[z^{\eta+1}-\left(z^{-}\right)^{\eta+1}\right]-\frac{\sigma}{2}\left[z^{2}-\left(z^{-}\right)^{2}\right] .
$$

Note that $\hat{g}(z)<0$ for $0 \leq z<z^{-}$.

Since the right-hand side of Eq. (46) is negative we must have $\left|d z / d \xi\left(\xi^{-}\right)\right|>|d z / d \xi(\xi)|$ for $\xi>\xi^{-}$.

We consider a wave moving to the right direction. Thus $z(\xi)$ is a decreasing function of $\xi$ and $z\left(\xi^{-}\right)=z^{-}>z(\xi)$ for $\xi>\xi^{-}$. In addition $\lim z(\xi)=0$. By letting $\xi$ tending to infinity in Eq. (46) we obtain

$$
\left(\frac{d z}{d \xi}(\xi)\right)^{2}=\frac{2 \mu}{\eta+1}\left(z^{-}\right)^{\eta+1}-\sigma\left(z^{-}\right)^{2} .
$$

Thus Eq. (46) reduces to

$$
\frac{1}{2}\left(\frac{d z}{d \xi}\right)^{2}=\widetilde{g}(z)
$$

with

$$
\widetilde{g}(z)=\frac{\mu}{\eta+1} z^{\eta+1}-\frac{\sigma}{2} z^{2}=\int_{0}^{z} g(u) d u .
$$

For $0<z<z^{-}$we have $g(z)>0$ and thus $\widetilde{g}(z) \geq 0$.

Considering that $\frac{d z}{d \xi}<0$ for a right moving wave, we have

$$
\frac{d z}{d \xi}=-\sqrt{2 \tilde{g}(z)}
$$

and by integration

$$
\xi=\xi^{-}+\int_{z}^{z^{-}} \frac{d u}{\sqrt{2 \widetilde{g}(u)}} .
$$

For $z>0$, the function $z \rightarrow 1 / \sqrt{\widetilde{g}(z)}$ is integrable in the vicinity of $z=0$ when $\eta<1$. This is due to the fact that for small values of $z$ the function $\widetilde{g}(z)$ is equivalent to $[\mu /(\eta+1)] z^{\eta+1}$.

Therefore the value $z=0$ is reached at $\xi^{+}<\infty$ defined by

$$
\xi^{+}=\xi^{-}+\int_{0}^{z^{-}} \frac{d u}{\sqrt{2 \widetilde{g}(u)}} .
$$

From Eq. (49) we have $\frac{d z}{d \xi}\left(\xi^{+}\right)=0$.

The integration of Eq. (52) can be made explicitly, see Appendix B. It is shown that

$$
\begin{aligned}
\xi= & \xi+\frac{2}{(1-\eta) \sqrt{\sigma}}\left[\arcsin \left(\sqrt{\frac{\sigma(\eta+1)}{2 \mu}}\left(z^{-}\right)^{(1-\eta) / 2}\right)\right. \\
& \left.-\arcsin \left(\sqrt{\frac{\sigma(\eta+1)}{2 \mu}} z^{(1-\eta) / 2}\right)\right]
\end{aligned}
$$

The shock-front structure is given by the function $z(\xi)$. This function is defined by the parametric representation $z \rightarrow \xi$ (54). Note that the shock front is not entirely described by considering only variation in $\xi$ on the interval $\xi^{-} \leq \xi \leq \xi^{+}$. By letting $\xi$ vary below the value $\xi^{-}$the totality of the shock front from $z=0$ to the maximum value of $z$ can be described.

A lower estimate of the shock width can be obtained by noting that $z^{+}=0$ at $\xi^{+}$and by using the definition (45) of $z^{-}$. An upper estimate is found by considering that about half of the leading front is spanned when $\xi$ varies from $\xi^{+}$to $\xi^{-}$,

$$
\begin{gathered}
w_{\text {lower }}=\xi^{+}-\xi^{-}=\frac{2}{(1-\eta) \sqrt{\sigma}} \arcsin \left(\sqrt{\frac{\eta+1}{2}}\right), \\
w_{\text {upper }}=2 w_{\text {lower }} .
\end{gathered}
$$

From Eq. (55) it follows that the shock width is not sensitive to the shock amplitude (i.e., to the impact velocity $v^{-}$). This point will be checked against direct numerical calculations. 
Let us remind that for a uniform bead chain and for a $(1: 1)$ dimer with uniform bead diameter $D, \sigma$ is scaled by $1 / D^{2}$, see Eq. (21). Therefore the shock width is scaled by the bead diameter $D$.

\section{COMPARISON OF ANALYTICAL, NUMERICAL, AND EXPERIMENTAL APPROACHES FOR A CHAIN OF DIMERS}

\section{A. Characterization of the state (-) behind the shock: consistency of analytical approaches}

In this manuscript we have evaluated the material state (-) far behind the shock front and the shock velocity following two different analytical approaches: (i) The first approach was developed in Sec. V and used the quasistatic homogeneized compression response of the chain of particles and the jump conditions across the shock. This approach could be applied to any periodic bead chain; (ii) The second approach was specifically developed for a chain of (1:1) dimers. It was based on the traveling wave Eq. (15), which is a continuous version of the dynamic response of the discrete bead system.

The results of the first approach (i) have been found to be in good agreement with numerical simulations. The consistency with the second approach (ii), which is specific to a (1:1) dimer, needs also to be evaluated.

The shock intensity can be characterized either by the impact velocity $v^{-}$or by the shock velocity $\hat{V}_{S}$ as $v^{-}$and $\hat{V}_{S}$ are in one-to-one correspondence. We consider here that the shock velocity $\hat{V}_{S}$ is given. Denote by $\lambda_{1}^{-}$and $\lambda_{2}^{-}$the stretches at state (-) determined by the approaches (i) and (ii), respectively. For consistency one should have $\lambda_{1}^{-}=\lambda_{2}^{-}$.

Using the approach (i) it follows from Eq. (41) that

$$
\left(1-\lambda_{1}^{-}\right)^{k-1}=\hat{V}_{S}^{2} \frac{\hat{\rho}_{0}}{K_{e f f}} .
$$

According to the approach (ii), the state (-) is defined by $z^{-}=(\mu / \sigma)^{1 /(1-\eta)}$. Combining with Eq. (20) we obtain

$$
\left(1-\lambda_{2}^{-}\right)^{(1-\eta) / p}=\frac{\mu}{\sigma}=V_{S}^{2} \frac{k p+a}{p+a},
$$

where the expressions (17) and (18) of $\sigma$ and $\mu$ have been used.

Dividing Eqs. (56) and (57), noting that $k-1=(1-\eta) / p$ from Eq. (16) and considering Eq. (12), it follows that

$$
\left(\frac{1-\lambda_{2}^{-}}{1-\lambda_{1}^{-}}\right)^{k-1}=\frac{K_{e f f}\left(m_{1}+m_{2}\right)}{2 \hat{\rho}_{0} k D^{k+1} A} \frac{k p+a}{p+a} .
$$

For a (1:1) dimer with uniform bead radius, we have

$$
\begin{gathered}
K_{e f f}=\frac{2}{3} \frac{E_{1} E_{2} D^{2}}{E_{2}\left(1-v_{1}^{2}\right)+E_{1}\left(1-v_{2}^{2}\right)}, \\
A=D^{-k} K_{e f f}, \\
\hat{\rho}_{0}=\frac{m_{1}+m_{2}}{2 D} .
\end{gathered}
$$

Thus, Eq. (58) can be rewritten as

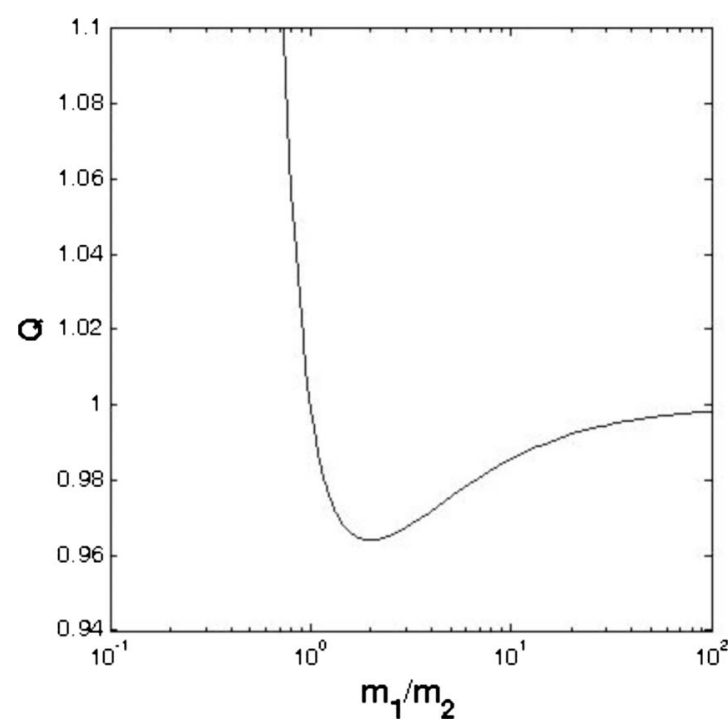

FIG. 3. Variation of the parameter $Q$, defined by Eq. (64), in terms of the mass ratio of particles. A value of $Q$ close to unity indicates a good consistency between the two analytical approaches used for characterizing the state behind the shock.

$$
\left(\frac{1-\lambda_{2}^{-}}{1-\lambda_{1}^{-}}\right)^{k-1}=\frac{k p+a}{k(p+a)} .
$$

Considering that $k=3 / 2$, Eq. (62) takes the form

$$
\left(\frac{1-\lambda_{2}^{-}}{1-\lambda_{1}^{-}}\right)^{1 / 2}=Q
$$

with

$$
Q=\frac{p+\frac{2}{3} a}{p+a} .
$$

For uniform beads, we have $\lambda_{1}^{-}=\lambda_{2}^{-}$since $a=0$, according to Eq. (25). Thus, the same state (-) is predicted by approaches (i) and (ii).

However for a (1:1) dimer the approaches (i) and (ii) do not provide in general exactly the same results. An estimate of the gap between the results can be obtained by comparing $Q$ with unity as shown in Fig. 3. From Eqs. (14) and (19) it appears that $a$ and $p$ are solely functions of $k$ and of the mass ratio $m_{1} / m_{2}$. As $k$ has the fixed value $3 / 2$, it is enough to show the variation of $Q$ in terms of $m_{1} / m_{2}$. Figure 3 shows that for $m_{1} / m_{2}$ larger than 0.8 , the maximum deviation of $Q$ from unity is about $4 \%$. For $m_{1} / m_{2}>2, Q$ is rapidly tending to the asymptotic value 1 . Thus to within few percent error, the two approaches are aimed to provide close results if one considers that the label (1) is affected to the heavy bead.

\section{B. Shock structure for a dimer bead chain: comparison of analytical, numerical, and experimental approaches}

It is worth evaluating the analytical results by comparing the shock structure given by numerical and experimental results with the predictions of approach (ii) developed in Sec. 

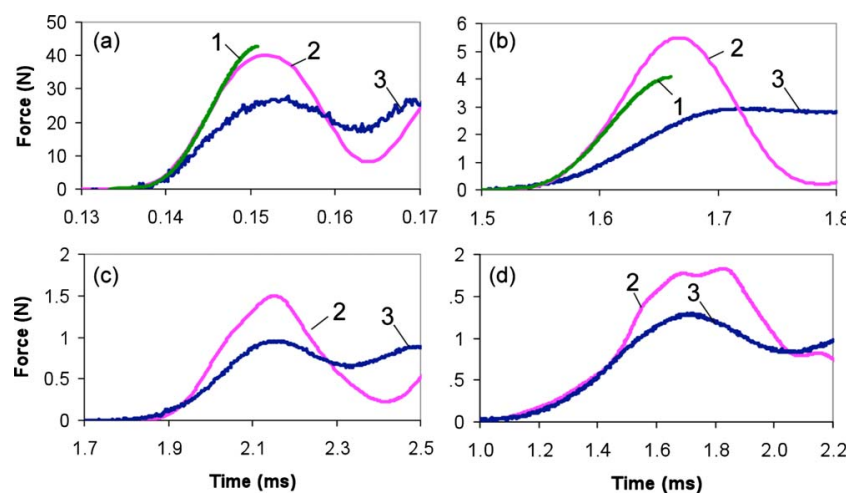

FIG. 4. (Color online) Comparison of analytical results from Eq. (54), (curve 1), numerical simulation of the discrete bead chain (curve 2) and experiments (curve 3). (a) Uniform chain composed of 53 particles. Striker velocity $0.44 \mathrm{~m} / \mathrm{s}$, curves correspond to the force-time response measured in particle 21; (b) (1:1) dimer chain composed of 53 particles. Striker velocity $0.44 \mathrm{~m} / \mathrm{s}$, curves correspond to particle 39; (c) (2:2) dimer chain, 64 particles total, striker velocity $0.2 \mathrm{~m} / \mathrm{s}$, curves correspond to particle 28; (d) (4:4) dimer chain 64 particles total, striker velocity $0.2 \mathrm{~m} / \mathrm{s}$ curves correspond to particle 26 .

VI and based on the long-wavelength approximation (15). Figure 4 shows the evolution of the force with time for different particles and dimer arrangements. The results predicted by the analytical approach (curve 1) are compared against numerical simulation (curve 2) and experimental measurements (curve 3). In the analytical approach, the spatial position $\xi$ is function of $z$ by Eq. (54), and of time through $t=\xi / \hat{V}_{S}+t_{0}$, where $t_{0}$ is an arbitrary time shift which can be calibrated to have the best match with the experimental results. Note that the result (54) was obtained from Eq. (15). Thus the long wave approximations, upon which this equation is based, provide a limitation of analytical results. Another difficulty in the analytical approach is to find an appropriate relationship between force and stretch during the transition between state $(+)$ and state $(-)$ where oscillations are observed. Using the homogenized quasistatic constitutive law (4) is not correct, except for states $(+)$ and (-) which are uniform and steady states. Considering the linear relationship between the force $F$ and the stretch $\lambda$ as described by the Rayleigh line

$$
F=\hat{\rho}_{0} \hat{V}_{S}^{2}(1-\lambda)=\hat{\rho}_{0} \hat{V}_{S}^{2} z^{p}
$$

will not be totally appropriate as this equation is obtained by using the macroscopic form of the conservation of momentum, Eq. (30), see Appendix C. Despite these limitations, Eq. (65) is used to derive the transient evolution of the compression force, to test the validity of the continuum approach by comparison against experimental measurements, Fig. 4.

The shock velocity $\hat{V}_{S}$ is calculated in terms of $v^{-}$with Eq. (41). The dependence of $F$ upon $\xi$ is described by the parametric representation $(\xi(z), F(z))$, where $\xi(z)$ is given by Eq. (54) and $F(z)$ by Eq. (65). Figure 4(a) shows that the shock front, predicted analytically for uniform bead chain, is in good correlation with numerical simulations. However, the correlation is not as good against experimental measurements. The presence of dissipative effects in experiments, which are neglected in the modeling, but cannot be avoided in the experiments, may explain this discrepancy [26]. The following estimates of the shock-front width are obtained from Eq. (55): $w_{\text {lower }}=8.3 \mathrm{~mm}, w_{\text {upper }}=16.6 \mathrm{~mm}$. The rise time at the shock front is evaluated as $\Delta t_{\text {lower }}=w_{\text {lower }} / \hat{V}_{S}$ $=0.013 \mathrm{~ms}, \Delta t_{\text {upper }}=0.026 \mathrm{~ms}$. These lower and upper estimates correlate well with the rise time estimated numerically and experimentally, see Fig. 4(a).

For the (1:1) dimer, from Eq. (55) we have, $w_{\text {lower }}$ $=13 \mathrm{~mm}, w_{\text {upper }}=26 \mathrm{~mm}, \Delta t_{\text {lower }}=0.119 \mathrm{~ms}$, and $\Delta t_{\text {upper }}$ $=0.238 \mathrm{~ms}$. The results of the lower $\left(\Delta t_{\text {lower }}\right)$ and upper bounds $\left(\Delta t_{\text {upper }}\right)$ of the rise time associated to the first peak provide an estimate for the temporal shock width of $\sim 0.12 \mathrm{~ms}$. This value is in good correlation with the temporal width observed in the fronts of Fig. 4(b). Figures 4(c) and $4(d)$ show the spatial distribution of the force along, respectively, (2:2) and (4:4) dimer chains for an impact velocity $v^{-}=0.2 \mathrm{~ms}^{-1}$. Here again, a good correlation could be found between estimations of the rise time by numerical and experimental results. In addition it was found that the width $w$ of the shock front scales with the cell size. For instance, for the (1:1) dimer chain the cell size is $2 D$ where $D$ $=4.76 \mathrm{~mm}$ is the bead diameter. It appears from the numerical results reported in Table II that $w \approx$ two cell sizes. It will be shown in the next section that the scaling law relating the width of the shock front to the cell size is verified for any kind of dimer configuration.

The numerical results presented in Fig. 4 are supported by experimental measurements. Indeed, it appears clearly in Fig. 4 that the rise times of the leading front (and consequently the widths of the shock front) obtained numerically are in good correlation with those obtained experimentally.

It is also important to note that the width of the steady shock front is almost independent upon the impact velocity $v^{-}$, see for example the results reported in Table II showing for a $(1: 1)$ dimer chain a quasi-invariance of $w$ in the range $0.1 \mathrm{~ms}^{-1} \leq v^{-} \leq 100 \mathrm{~ms}^{-1}$. The analytical estimates, from Eq. (55), confirm the invariance of $w$ with respect to $v^{-}$.

\section{RELATIONSHIP BETWEEN THE CELL SIZE AND THE WIDTH OF THE STEADY SHOCK FRONT}

The results of the previous section (scaling law of the width $w$ of the shock front and independence of $w$ with respect to the impact velocity) obtained for uniform bead chains and for $(1: 1)$ dimer chains are extended here for unit cells of any size and any configuration. The analytical approach developed in Sec. VI does not apply here, since it is not proved that Eq. (15) remains valid when the unit cell contains more than two beads. Therefore the shock-width is evaluated from numerical simulations of the dynamic response of the discrete bead chain.

The numerical evaluation of the shock width is made in the following way. At a given bead, the analysis of the time history of the force provides an evaluation of the arrival time of the shock front $\left(t_{\text {arrival }}\right)$ and of the time at which the maximum of the force is reached $\left(t_{\max }\right)$. However the time differ- 

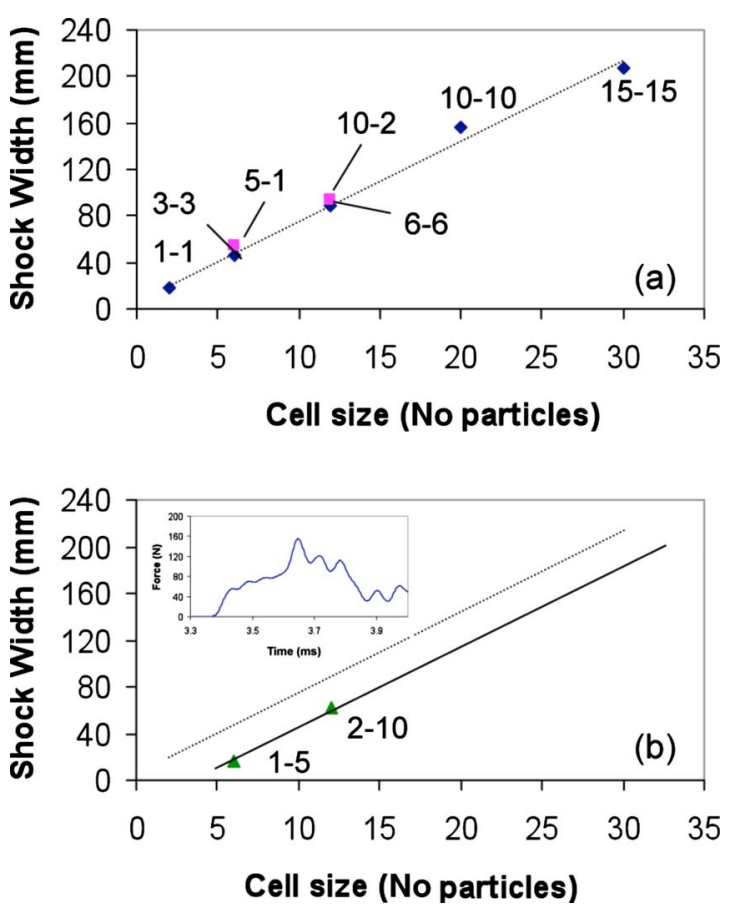

FIG. 5. (Color online) Variation in the shock width as a function of the cell size for different configurations of particle chains. (a) Response of dimers in which steel beads represent the predominant mass contribution; (b) response for dimers in which the number of PTFE beads in a unit cell prevails over the number of steel beads. The dotted line reports the linear fitting of the data in panel (a) for comparison. The inset shows the force-time relation for the disordered shock front in a $(2: 10)$ dimer impacted by a steel particle with velocity $10 \mathrm{~m} / \mathrm{s}$. The curve represents the numerical result obtained in particle 116.

ence $\Delta t=t_{\text {arrival }}-t_{\max }$ depends naturally on the location of the bead (inside the unit cell) where time history is recorded. Taking the average of $\Delta t$ over the whole set of beads of a unit cell provides a quantity $\Delta t_{\text {average }}$-which, under steady wave propagation, will be time independent. Finally, an estimate of the shock-front width is given by $\hat{V}_{S} \Delta t_{\text {average }}$. This characterization of the shock width may be rather computationally lengthy when large cell sizes are considered. It has been checked that an easier way to proceed, providing close results, consists in evaluating $\Delta t$ at the bead in the middle (or closest to the middle) of the soft phase of the unit cell considered.

In Fig. 5, the shock-front width, evaluated as above, is reported in terms of the cell size for various cell configurations. The shock width is independent of the impact velocities and has been obtained by averaging runs between $(0.1-100 \mathrm{~m} / \mathrm{s})$. From Fig. 5(a), it appears that the shock width is proportional to the cell size for $\left(N_{1}, N_{2}\right)$ dimers with $N_{1} \geq N_{2}$. For $N_{1}<N_{2}$, an affine relationship is found, which is slightly different from the linear law obtained for $N_{1} \geq N_{2}$. We do not have a clear explanation of this difference. However, in all cases the shock-front width can be considered as scaled by the cell size. These results extend those of Sec. VII obtained for uniform chain and (1:1) dimers.
The invariance with respect to the impact velocity of the shock-front width, demonstrated in Sec. VII for uniform chain and $(1: 1)$ dimers, is extended here by considering various cell configurations. The results of Table III for a $(5: 1)$ dimer provide an illustration of this invariance in the range of impact velocities $0.1 \mathrm{~ms}^{-1} \leq v^{-} \leq 100 \mathrm{~ms}^{-1}$. Indeed, for all our simulations made for various cell configurations, the same invariance was found.

It is interesting to compare the dynamic response of a composite bead chain with the response of layered structures. Zhuang et al. [9] considered periodically layered composites made of a succession of soft (polymeric) and hard (metallic) layers.

In their shock experiments the laminates were deformed plastically and it was found that the stress jump $\Delta \sigma$ across the shock was related to the maximum strain rate within the shock $|\dot{\varepsilon}|_{\max }$ by a power-law of the form

$$
|\dot{\varepsilon}|_{\max }=B(\Delta \sigma)^{h_{L}} .
$$

For the laminates analyzed by Zhuang et al. [9] the exponent was in the range: $1.6<h_{L}<2.4$. These results were interpreted theoretically by Molinari and Ravichandran [43] in terms of gradient plasticity approaches. A power-law dependence of the form (66) seems to be a general feature in shock experiments, the exponent $h$ depending on the material considered. Swegle and Grady [39] discussed the relationship (66) in the context of single-phase metallic materials. They found from shock-wave experiments that Eq. (66) was fairly well satisfied for various metals with the exponent $h$ having the "universal" value $h_{S G}=4$. Swegle and Grady [39] interpreted these results in terms of a material strain-rate sensitivity parameter $m$. According to the interpretation of experimental results given in [39], single-phase metals should have the same rate sensitivity $m=0.5$ when subjected to the high strain rates undergone within the shock layer. These results were revisited by Molinari and Ravichandran [44] who found that, within the shock layer, $m$ could be different from 0.5 , although remaining close to this value.

The composite bead chains considered in this paper represent highly nonlinear material systems for which it is worth to characterize the "shock" response as done previously for solid continuum by Swegle and Grady [39], and Molinari and Ravichandran $[43,44]$. A way to characterize the material response within the shock is to analyze the relationship between load (compressive stress or force) and strain rate. With respect to the approach developed by Swegle and Grady [39] the stress $\sigma$ has to be replaced here by the force $F$. Since $F^{+}=0$ (state of rest ahead of the shock), the variation in the force between state $(+)$ and state $(-)$ is: $\Delta F=F^{-}$.

We define an average strain rate $\overline{\dot{\varepsilon}}$ within the shock front as follows. When a particle crosses the leading shock front the corresponding compressive force is raised from 0 to $F_{\text {max }}$, which is about two times $F^{-}$. The time durations to reach $F_{\max }$ and $F^{-}$are, respectively, about $w / \hat{V}_{S}$ and $(w / 2) / \hat{V}_{S}$. The increasing of strain (in absolute value) corresponding to the variation of $F$ from 0 to $F^{-}$is $\left|\ln \lambda^{-}\right|$. Therefore $\overline{\dot{\varepsilon}}$ can be defined as 


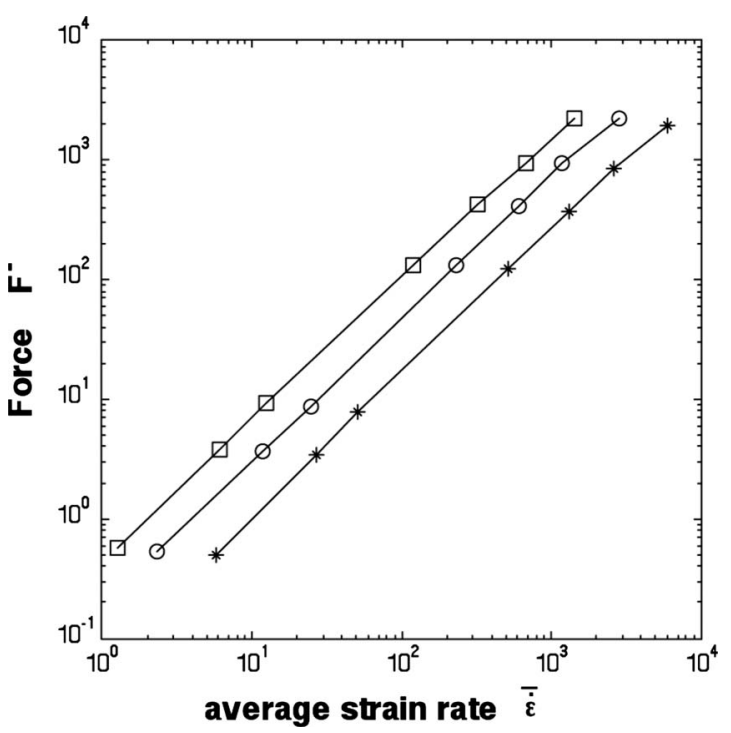

FIG. 6. Force $F^{-}$versus average strain rate $\overline{\dot{\varepsilon}}$ within the shock layer for various cell sizes: (1:1) dimer chains (crosses); (3:3) (circles); (6:6) (squares).

$$
\overline{\dot{\varepsilon}}=\frac{\left|\ln \lambda^{-}\right|}{(w / 2) / \hat{V}_{S}} .
$$

Considering that for moderate compression we have $\left|\ln \lambda^{-}\right|$ $\approx 1-\lambda^{-}$, it can be checked by using Eq. (41) that

$$
\overline{\dot{\varepsilon}} \approx 2 \frac{v^{-}}{w} .
$$

Figure 6 shows the dependence of $F^{-}$in terms of $\overline{\dot{\varepsilon}}$ in a $\log$-log diagram. Different material configurations are considered (1:1), (3:3), and (6:6) dimers. Note that the cells (3:3) and (6:6) are obtained from the (1:1) dimer's cell by multiplying the number of beads, respectively, by a factor $\gamma=3$ and $\gamma=6$. For a given unit-cell configuration, the linear relationship obtained in Fig. 6 indicates that a power-law dependence of the form applies,

$$
\overline{\dot{\varepsilon}}=B\left(F^{-}\right)^{h_{B C}}
$$

where the subscript (BC) on the exponent holds for bead chain.

As the slopes of the different lines in Fig. 6 are the same, it can be concluded that the exponent has a constant value independent of the ratio $\gamma$. This value, estimated as $h_{B C}$ $=0.83 \pm 0.009$ is in close agreement with the theoretical value $h_{\text {theor }}=0.833$, as predicted below for $k=3 / 2$ by Eq. (71).

Analytical results can be obtained by combining Eqs. (68) and (40) into

$$
F^{-}=b(\overline{\dot{\varepsilon}})^{1 / h_{\text {theor }}}
$$

with

$$
b=K_{e f f}\left(\frac{\hat{\rho}_{0}}{K_{e f f}}\right)^{k /(1+k)} w^{2 k /(1+k)}, \quad h_{\text {theor }}=\frac{1+k}{2 k} .
$$

Since for a given bead chain, the shock width was shown to be almost independent of the impact velocity $v^{-}$, we have, by comparison with Eq. (69): $h_{B C}=(1+k) / 2 k$.

Equations (70) and (71) show that for a given value of $F^{-}$ (i.e., for a given shock strength), the average strain rate $\overline{\dot{\varepsilon}}$ within the shock front is nearly inversely proportional to the shock width $w$. As $w$ is proportional to the cell size, it can be concluded that $\overline{\dot{\varepsilon}}$ is inversely proportional to the cell size. Indeed, in the log-log diagram represented in Fig. 6, the curve associated to the $(1: 1)$ dimer is obtained from that associated to the $(3: 3)$ dimer by a horizontal shift of $\ln (\gamma)$, where $\gamma=3$ is the ratio between cell sizes.

It should be noted that the slope of the lines in Fig. 6 depends solely upon the value of the exponent $k$ in the Hertz constant law. However, for a given composition of the dimer chain, the position of the corresponding line in Fig. 6 is given by the coefficient $b$ defined in Eq. (71). For a $(1: 1)$ dimer chain, this coefficient depends of the composition of the unit cell via $K_{\text {eff }}$ and $\hat{\rho}_{0}$. The coefficient $b$ depends also from $\eta=\eta\left(k, m_{1} / m_{2}\right)$ and $\sigma=\frac{1}{D^{2}} f\left(k, m_{1} / m_{2}\right)$ through the estimates $(55)$ of the shock-front width.

The relationship (70) can be viewed as representative of the material response across the shock. It is worth noting that the force $F^{-}$exhibits a strain-rate dependence that does not stem from the quasistatic response of the bead chain as the beads are assumed to have a purely elastic response. From Eq. (70) it appears that the parameter $b$ vanishes when inertia is neglected $\left(\hat{\rho}_{0}=0\right)$. Thus the rate dependence of $F^{-}$is clearly the consequence of inertia effects.

The exponent $h$ varies within a broad range depending on the structure of the material considered and on the physical mechanisms that control the shock front. The largest value $h=4$ is obtained for single-phase metals and is due to dissipation. The lowest value $h_{B C}=0.833$ is for the highly nonlinear bead chains considered in this paper. Intermediate values of the exponent $1.6<h_{L}<2.4$ are found for continuum layered materials.

It is worth noting that $h_{B C}$ is solely dependent upon the exponent $k=3 / 2$ of the Hertz contact law, which is only dependent on the geometry of the contact. In contrast for continuum such as polycrystalline metals and layered composites, the exponent $h$ is highly dependent on material properties. However, for bead chains, material properties do have an effect on the level of strain rate through the prefactor $b$ in the law (70).

It is also important to note that a low value of $h$ is beneficial. Indeed, in that case, we have a weak dependence of the strain rate (within the shock front) with respect to the pressure loading.

\section{CONCLUSIONS}

We have considered quasistationary shock waves propagating in homogeneous and heterogeneous chains of spherical particles generated by the impact of a massive striker. The chains of particles were composed by the periodic rep- 
etition of a basic unit cell composed of an arrangement of two types of beads (steel and polytetrafluoroethylene). The analysis was based on direct numerical simulations of the dynamic response of the discrete bead system and on analytical results obtained from homogenized equations. Theoretical and numerical results were also compared against experimental tests and a good correlation was found with respect to the material state behind the shock.

For the amplitude of the leading hump in the shock front, a qualitative agreement was found with respect to experimental data. However a quantitative agreement could not be reached as dissipative effects in experiments produced an important damping (particularly when polymeric beads were involved), while the modeling was developed for purely elastic beads (no form of dissipation was included).

It was also theoretically shown that in the absence of dissipation dispersive effects structured the leading front of the shock wave. Two types of dispersive effects are involved in the problem: classical lattice dispersion found in any discrete system of punctual masses with interacting elastic potentials, and dispersion due to the impedance mismatch between material domains. Although dissipative effects are present in experiments, the measured shock-front width was found to be in good agreement with the one predicted by theory and by discrete numerical analysis (that did not account for dissipation).

In addition, we demonstrated by numerical, analytical, and experimental means that the width of the shock front is scaled by the size of the unit cell of the composite chain and is independent upon the impact velocity. A power-law relationship was found between the average strain rate within the shock front and the jump of the compressive load across the shock. This result is similar to theoretical and experimental laws obtained for continuum materials (polycrystalline metals and layered composites). The power law is characterized by two parameters, an exponent $h$ and a prefactor $B$. Interestingly, for a bead chain, $h$ is not affected by material properties but is solely dependent upon the geometry of the contact between beads. Indeed, $h$ has a value quite different from those corresponding to continuum materials. This confers to bead chains the unique feature of having a strain rate within the leading shock front with a weaker dependence upon the impact velocity than for other materials. However, the prefactor $B$ is a function of material properties and so is the width of the shock front. This leaves place for optimizing the internal structure of a composite chain of particles with the perspective of decreasing the level of strain rates generated by the impact of a projectile, suggesting these systems as effective novel energy dispersive materials.

\section{ACKNOWLEDGMENTS}

C.D. acknowledges the Army Research Office (Proposal No. 54272-EG) and the NSF CAREER for support of this work. C.D. thanks the University of Metz for hospitality during her visit. We also thank Dr. Mason Porter for providing the program code for the numerical calculations.

\section{APPENDIX A: THE OVERALL EFFECTIVE RESPONSE OF A COMPOSITE BEAD CHAIN UNDER QUASISTATIC COMPRESSIVE LOADING}

We consider a composite chain of particles as defined in Sec. II. The numbers of beads of type (1) and (2) per unit cell are, respectively, denoted by $N_{1}$ and $N_{2}$. The compressive force $F$ is applied quasistatically. The resulting overall stretch is $\lambda$. The relationship $\lambda \rightarrow F$ (effective response of the composite bead chain) is determined here in general while it was only given in Sec. II for a $(1: 1) \operatorname{dimer}\left(N_{1}=1\right.$ and $N_{2}$ $=1$ ).

Consider two adjacent beads (i) and (j) with centers $A_{i}$ and $A_{j}$, respectively. The bead type is defined by the value of $i$ or $j$ [for instance $i=1$ means that the first bead is of type (1)]. Under the compressive force $F$, the stretch undergone by the segment $L_{i j}=A_{i} A_{j}$ is denoted by $\lambda_{i j}$. The initial distance between the centers is $A_{i} A_{j}=\left(D_{i}+D_{j}\right) / 2$.

It is easily checked that an unit cell can be defined by the following sequence: $N_{1}-1$ segments $L_{11}$ with stretch $\lambda_{11}$, a segment of type $L_{12}$ with stretch $\lambda_{12}, N_{2}-1$ segments of type $L_{22}$ with stretch $\lambda_{22}$, and finally a segment of type $L_{21}$ with stretch $\lambda_{21}$.

The stretches $\lambda_{12}$ and $\lambda_{21}$ resulting from the contact of beads (1) and (2) are identical and are given by Eqs. (3a) and (3b),

$$
\begin{gathered}
\lambda_{12}=\lambda_{21}=1-\left(\frac{F}{K_{12}}\right)^{1 / k}, \\
K_{12}=\frac{1}{3} E^{*} \sqrt{D_{1} D_{2}}\left(D_{1}+D_{2}\right), \\
\frac{1}{E^{*}}=\frac{1-\nu_{1}^{2}}{E_{1}}+\frac{1-\nu_{2}^{2}}{E_{2}} .
\end{gathered}
$$

The stretch $\lambda_{11}$ corresponds to the contact of two identical beads of type (1). The result follows from the relationships (A1)-(A3) where the subscript 2 is replaced by 1 ,

$$
\begin{gathered}
\lambda_{11}=1-\left(\frac{F}{K_{11}}\right)^{1 / k}, \\
K_{11}=\frac{2}{3} E^{*} D_{1}^{2}, \\
\frac{1}{E^{*}}=2 \frac{1-\nu_{1}^{2}}{E_{1}} .
\end{gathered}
$$

The stretch $\lambda_{22}$ is given by Eqs. (A4)-(A6) with $1 \rightarrow 2$.

The overall stretch (or effective stretch) in the composite bead chain is obtained as the weighted average of the local stretches $\lambda_{11}, \lambda_{22}$, and $\lambda_{12}$,

$$
\begin{gathered}
\lambda=f_{11} \lambda_{11}+f_{22} \lambda_{22}+f_{12} \lambda_{12}, \\
f_{11}=\frac{\left(N_{1}-1\right) D_{1}}{N_{1} D_{1}+N_{2} D_{2}}, \quad f_{22}=\frac{\left(N_{2}-1\right) D_{2}}{N_{1} D_{1}+N_{2} D_{2}},
\end{gathered}
$$




$$
f_{12}=\frac{D_{1}+D_{2}}{N_{1} D_{1}+N_{2} D_{2}} .
$$

Note that

$$
f_{11}+f_{22}+f_{12}=1 \text {. }
$$

From Eqs. (A1) and (A4) and the relationship for $\lambda_{22}$ similar to Eq. (A4), we have

$$
\left(K_{12}\right)^{1 / k}\left(1-\lambda_{12}\right)=\left(K_{11}\right)^{1 / k}\left(1-\lambda_{11}\right)=\left(K_{22}\right)^{1 / k}\left(1-\lambda_{22}\right) .
$$

Using Eqs. (A7) and (A9), it follows that

$$
1-\lambda=f_{11}\left(1-\lambda_{11}\right)+f_{22}\left(1-\lambda_{22}\right)+f_{12}\left(1-\lambda_{12}\right),
$$

which by Eq. (A10) can be written as

$$
1-\lambda=A\left(1-\lambda_{11}\right),
$$

with $A=f_{11}+f_{22}\left(K_{11} / K_{22}\right)^{1 / k}+f_{12}\left(K_{11} / K_{12}\right)^{1 / k}$.

Thus, by elimination of $\lambda_{11}$ between Eqs. (A4) and (A12) we obtain the effective response of the composite bead chain under quasistatic compression,

$$
F=F_{Q S}(\lambda)=K_{\text {eff }}(1-\lambda)^{k}
$$

with

$$
K_{e f f}=\left(f_{11}\left(K_{11}\right)^{-1 / k}+f_{22}\left(K_{22}\right)^{-1 / k}+f_{12}\left(K_{12}\right)^{-1 / k}\right)^{-k} .
$$

The stiffness parameters $K_{11}, K_{22}$, and $K_{12}$ have been calculated in the case of Hertzian contact, see Eqs. (A2) and (A5). However, the result (A14) remains valid for non-Hertzian contact, with appropriate definitions of $K_{11}, K_{22}$, and $K_{12}$.

\section{APPENDIX B: INTEGRATION OF THE SHOCK PROFILE}

Integration of Eq. (52) (providing the shock structure) is obtained as follows: consider the integral,

$$
I=\int \frac{d z}{\sqrt{2 \widetilde{g}(z)}}=\frac{1}{\sqrt{\sigma}} \int \frac{d z}{\sqrt{a_{1} z^{\eta+1}-z^{2}}},
$$

where $a_{1}=2 \mu /(\eta+1) \sigma$.

With the change of variable $z=a_{1}^{1 /(1-\eta)} X^{2 /(1-\eta)}$, Eq. (B1) can be written as

$$
\begin{aligned}
I & =\frac{2}{(1-\eta) \sqrt{\sigma}} \int \frac{d X}{\sqrt{1-X^{2}}} \\
& =\frac{2}{(1-\eta) \sqrt{\sigma}} \arcsin \left(\sqrt{\frac{\sigma(1+\eta)}{2 \mu}} z^{(1-\eta) / 2}\right)+\text { const } .
\end{aligned}
$$

\section{APPENDIX C: RAYLEIGH LINE}

From the usual formulation of traveling waves Eqs. (30) and (32) can be expressed in terms of the variable $\xi=x$ $-\hat{V}_{S} t$ and integrated between state (+) and the current state,

$$
F-F^{+}=\hat{\rho}_{0} \hat{V}_{S}\left(v-v^{+}\right)
$$

$$
v-v^{+}=-\hat{V}_{S}\left(\lambda-\lambda^{+}\right) .
$$

Considering that $F^{+}=0$ and $\lambda^{+}=1$, the following relationship is obtained:

$$
F=\hat{\rho}_{0} \hat{V}_{S}^{2}(1-\lambda)=\hat{\rho}_{0} \hat{V}_{S}^{2} z^{p},
$$

which defines the Rayleigh line.
[1] G. B. Whitham, Linear and Nonlinear Waves (Pure and Applied Mathematics) (Wiley, New York, 1999).

[2] M. Remoissenet, Waves Called Solitons (Concepts and Experiments), 3rd ed. (Springer-Verlag, Berlin, 1999).

[3] V. F. Nesterenko, V. M. Fomin, and P. A. Cheskidov, in Attenuation of Strong Shock Waves in Laminate Materials, edited by J. E. U. Nigul, IUTAM Symposium on Non-Linear Deformation Waves (Springer-Verlag, Berlin, 1983), pp. 191-197.

[4] C. T. Sun, J. D. Achenbach, and G. Herrmann, ASME J. Appl. Mech. 35, 467 (1968).

[5] J. D. Achenbach, in A Theory of Elasticity with Microstructure for Directionally Reinforced Composites, International Center for Mechanical Sciences (Springer-Verlag, New York, 1973), Vol. 167.

[6] R. M. Christensen, Mechanics of Composite Materials (Wiley, New York, 1979).

[7] C. Boutin, Int. J. Solids Struct. 33, 1023 (1996).

[8] I. A. Kunin, Elastic Media with Microstructure (SpringerVerlag, Berlin, 1983), Vols. 1 and 2.

[9] S. M. Zhuang, G. Ravichandran, and D. E. Grady, J. Mech.
Phys. Solids 51, 245 (2003).

[10] V. F. Nesterenko, J Appl. Mech. Tech. Phys. 24, 733 (1984); Prikl. Mekh. Tehk. Fiz. 24, 136 (1983).

[11] V. F. Nesterenko, Dynamics of Heterogeneous Materials (Springer-Verlag, New York, 2001).

[12] M. A. Porter et al., Phys. Rev. E 77, 015601 (2008).

[13] M. A. Porter et al., Physica D 238, 666 (2009).

[14] S. Sen, J. Hong, J. Bang, E. Avalosa, and R. Doney, Phys. Rep. 462, 21 (2008).

[15] C. Daraio et al., Phys. Rev. Lett. 96, 058002 (2006).

[16] R. L. Doney and S. Sen, Phys. Rev. E 72, 041304 (2005).

[17] J. Hong, Phys. Rev. Lett. 94, 108001 (2005).

[18] V. F. Nesterenko et al., Phys. Rev. Lett. 95, 158702 (2005).

[19] C. Daraio et al., Phys. Rev. E 73, 026610 (2006).

[20] C. Coste, E. Falcon, and S. Fauve, Phys. Rev. E 56, 6104 (1997).

[21] C. Coste and B. Gilles, Eur. Phys. J. B 7, 155 (1999).

[22] C. Daraio and V. F. Nesterenko, Phys. Rev. E 73, 026612 (2006).

[23] C. Daraio et al., Phys. Rev. E 72, 016603 (2005). 
[24] E. Hascoet and H. J. Herrmann, Eur. Phys. J. B 14, 183 (2000).

[25] E. Hascoet, H. J. Herrmann, and V. Loreto, Phys. Rev. E 59, 3202 (1999).

[26] E. B. Herbold and V. F. Nesterenko, Phys. Rev. E 75, 021304 (2007).

[27] E. B. Herbold and V. F. Nesterenko, Appl. Phys. Lett. 90, 261902 (2007).

[28] V. F. Nesterenko et al., J. Acoust. Soc. Am. 123, 3271 (2008).

[29] A. Rosas and K. Lindenberg, Phys. Rev. E 69, 037601 (2004).

[30] S. Job et al., Granular Matter 10, 13 (2007).

[31] I. N. Sneddon, Int. J. Eng. Sci. 3, 47 (1965).

[32] Fraternali, F., M. A. Porter, and C. Daraio, Mechanics of Advanced Materials and Structures (to be published).

[33] R. Manvi and G. E. Duvall, Br. J. Appl. Phys., J. Phys. D 2 , 1389 (1969).

[34] R. Manvi, G. E. Duvall, and S. C. Lowell, J. Appl. Phys. 40, 3771 (1969).

[35] B. E. McDonald, J. Acoust. Soc. Am. 120, 3503 (2006).
[36] D. H. Tsai and C. W. Beckett, J. Geophys. Res. 71, 2601 (1966).

[37] T. G. Hill and L. Knopoff, J. Geophys. Res. 85, 7025 (1980).

[38] M. H. Rice, R. G. McQueen, and J. M. Walsh, in Solid State Physics, edited by F. Seitz and F. S. A. D. Turnbull (Academic, New York, 1958), Vol. 6.

[39] J. W. Swegle and D. E. Grady, J. Appl. Phys. 58, 692 (1985).

[40] D. J. Korteweg and G. de Vries, Edinburgh and Dublin Philosophical Magazine and Journal of Science 5, 422 (1895).

[41] C. D. Lundergan and D. S. Drumheller, in Dispersion of Shock Waves in Composite materials, edited by J. J. Burke and V. Weiss, Shock Waves and the Mechanical Properties of Solids (Syracuse University Press, New York, 1971).

[42] D. Grady, J. Mech. Phys. Solids 46, 2017 (1998).

[43] A. Molinari and R. Ravichandran, J. Mech. Phys. Solids 54, 2495 (2006).

[44] A. Molinari and R. Ravichandran, J. Appl. Phys. 95, 1718 (2004). 